\title{
Image Perception of Female Breast Beauty and Its Relation to 3D Anthropometric Measurements
}

\author{
Chao Sun, Rong Zheng*, Jian Li, Haiqiao Huang, Minghai Cui \\ Aimer Human Engineering Research Centre, Beijing Institute of Fashion Technology \\ Beijing 100029, China
}

\begin{abstract}
This study aimed to explore the relationship between breast shape measurements and breast beauty assessment. Breast Beauty Subjective Assessment System (BBSAS) was developed to evaluate the beauty of breasts. 75 female subjects aged 20-39 years were selected for assessment from the anthropometric database of BIFT-Aimer Human Engineering Research Centre. 43 females working in intimate apparel design were chosen as judges to answer a five-scale questionnaire using the BBSAS. 66 detailed measurements especially related to the breast region were also taken for investigation. Data analysis results indicated that the breast beauty score was highly correlated with breast side view profile and breast erectness. Beautiful breasts selected by judges had the following common characteristics: the ratio of bust height to whole body height was 0.71; a triangle was constructed by front neck point, right bust point and left bust point was an isosceles triangle with the top angle less than $60^{\circ}$. The regression equation to predict the breast beauty scale was obtained based on key breast measurements. This study provides a new knowledge on relationship between the breast shape and breast beauty, as well as some useful guidelines for bra design.
\end{abstract}

Keywords: Breast Beauty; Beauty Subjective Assessment; 3D Anthropometric Measurements; Regression Analysis

\section{Introduction}

Female breast shape is a key constituent of their gender features, and vital for the beauty of female appearance. Fully understanding female breast structure is of great significance for the structure design of lingerie products and breast shaping for the mannequin development. Since breast shape, position, contour and volume can all directly influence the clothing design, parameterization study of breast shape beauty has become a key research direction in this field.

Since 1960s, Japanese Wacoal Corp. has carried out systematic studies to analyze the features of Japanese female body shapes, and published related research achievements on criteria of female body shape beauty in the past 30 years [1]. Gold Proportion, proposed in 1965, was formed on

${ }^{*}$ Corresponding author.

Email address: yuki.zheng@gmail.com (Rong Zheng). 
the basis of anthropometric measurements and questionaire survey on the ideal body shapes. The results indicated that if the body height was 100, the gold proportion of the breast, waist and hip girth should be 53:37:55. Beautiful Proportion, proposed in 1979 by Wacoal, introduced the age factor into the Gold Proportion, and established proportion formulas on measurements of such items as breast girth, waist girth, hip girth and leg length in proportion to the body height. Gold Canon, proposed in 1994 by Wacoal, summarized common characteristics of beautiful body shapes based on anthropometric measurements and subjective assessment, and proposed a new beauty index "Beauty Balance", including the girth balance of bust, waist \& hip, the torso proportion balance, three-dimensional balance of the breast, three-dimensional balance of the hip, the balance of height and weight, balance of height and proportion, etc.

Liang et al. categorized breast shapes into 9 basic types depending on 254 subjects and 10 3D measurement and took the intermediate type as the standard type [2]. It was concluded that the proportion of breast height to body height for young Chinese females was about 0.71 , and the ratio of distance between two bust points to the chest width was around 0.5. The distance between two bust points and height of the bust point were regarded as major factors influencing the female breast shape beauty. In addition, the relevant measurements of the breast cross section, such as ratio of chest width to distance between two bust points, ratio of bust girth to underbust girth and ratio of breast girth to distance between two bust points, were also used to describe the girth characteristics of the breast, and were taken as the basis to categorize breast types, together with the girth measurements [3].

Liu et al. defined the bust girth more than $54 \%$ of the body height as the beautiful breast [4]. Zhang summarized several characteristics necessary for the beautiful breast: distance between two bust points should not be more than $20 \mathrm{~cm}$, width of the breast base should be around 10-12 $\mathrm{cm}$, the height from the breast base to the bust point be around 5-6 cm, etc. [5]. Fang and Jiang discussed the aesthetic significance of the breast from the perspective of the physiological structure and shape categories of the breast, and confirmed the measurements related to the ideal breast judgment, including distance between two bust points, height of the breast, diameter of the areola, etc. [6]. Hou introduced a set of multiple regression equations to predict responses about bust height, breast position and shape using several predictors like weight, whole body height and hip girth [7].

Based on the breast shape and its position, Chen et al. carried out manual measurements for 100 female college students and obtained the breast-related data, and proposed a method to determine the position of the bust point through the measurements such as bust girth, distance between two bust points, the vertical distance from the centre of the nipple to the horizontal level of the central point of the upper arm on the same side. It was proposed that the perfect breast shape should meet the following requirements: the triangle constructed by front neck point, right bust point and left bust point should be an equilateral triangle. The ratio of the distance between the bust point and the outer-most point of the breast to the distance between the bust point and the inner-most point of the breast should be close to the gold proportion of 0.618 [8].

The physiological structure of the breast is also critical for the breast shape. Westreich et al. proposed that only the breast that is not drooping can be regarded as the perfect breast. They defined the landmarks for breast measuring according to bone points, and tried to avoid using soft tissues. Taking those points as references, they measured 50 white females who possessed perfect breasts. Based on data analysis results, they introduced the multiple regression equation to calculate the "standard" breast volume: first to multiply logarithm of the distance from the 
front neck point to the centre of the nipple (M-Ni) by 1.103, and to multiply logarithm of the distance between two nipples $(\mathrm{N}-\mathrm{Ni})$ by 0.811 ; then logarithm of the breast volume was obtained by adding up the previous two items [9].

Catanuto et al. obtained data about the breast and acquired base shapes of breasts through the 3D scanner [10]. They proposed a definition to distinguish different parts of female breast surface through measurements such as angles and curvature, so as to analyze the shape difference between the natural breast and the molded one.

In the field of painting and sculpture, usually the specific shape data or height ratios are used to describe the breast [11]. The judging standard for breast shape beauty is defined as the angle of the central axis between two breasts should be appropriate and lie on the spine [12]. Dan thought the breast that was located between the second rib and the sixth rib and also between the side edge of the breastbone and the front line of the armpit as conforming to the physiological and aesthetic beauty concept [13].

In spite of lots of researches on female breast shape beauty were carried out by domestic and overseas scholars, most of the researches focused on the extracting and analysis of the data on the breast surface shape, while the objective description and analysis of smoothness of the breast surface curves and the varying range of curvature is few, and the precedent research integrating the subjective assessment with objective measurements is also scarcely seen. Therefore, this study mainly focuses on investigating the relationship between the subjective results of breast beauty assessment and the breast anthropometric measurements. The aim is to quantitatively analyze the breast shape beauty so as to better understand the female breast beauty and provide the effective guide for the design and development of the lingerie products.

\section{Methodology}

\subsection{Judges and Assessed Samples}

Furnham et al. do not regard gender as the dominant factor affecting assessment of female body beauty [14-16]. In contrast, Fallon et al. indicated that there was a difference between the male judge and the female judge in assessing female body shape beauty and attractiveness [17-18]. In addition, factors such as appearance, complexion, hairstyle and temperament could all affect the judge's assessment of breast shape beauty and the whole body shape beauty.

Given that one of the major application directions of the breast shape beauty study is on research and development of the lingerie mannequin and bras, this study selected 43 female employees in underwear enterprises as judges, of whom 19 persons were underwear designers, 12 judges were craft-persons, and 12 judges were market sales persons. All of them were equipped with years of experience on research and development of underwear products.

In order to more objectively and effectively assess the breast shape beauty, this paper used 3D data to avoid interference of the outer factors. The procedures to determine samples for assessment are as follows: 1) considering that females aged from 20 to 39 are the major purchasing group for lingerie products, therefore the body samples of 456 subjects falling into this group were taken from the anthropometric database of BIFT-Aimer Human Engineering Research Centre. 2) in light of FZ/T 73012-2004 [19] and FZ/T 73011-2004 [20], the industry size and type standards for bras and briefs, the 456 body samples were categorized into different groups, as shown in Fig. 1, 
and 25 groups of bra/briefs sizes were obtained. " $\bigcirc$ " means there are matching samples in the specific group; 3) with consideration of factors like time, cost and coverage, in order to improve efficiency of the study, within the 25 groups, the Euclidean Distance Method was adopted. Taking four measurements (underbust girth, the difference between bust girth and underbust girth, waist girth and hip girth) that are used to determine bra/briefs sizes as key measurements item sizes of the four measurements item of each subject were obtained and the difference between each specific size was calculated together with the central value of each corresponding size group. Three sample bodies, respectively closest to the central value, farthest from the central value and right in the half way from the central value in each group, were selected to represent the corresponding groups. Hence, 75 subjects were chosen as samples for the breast shape beauty assessment.

Selected bra sizes

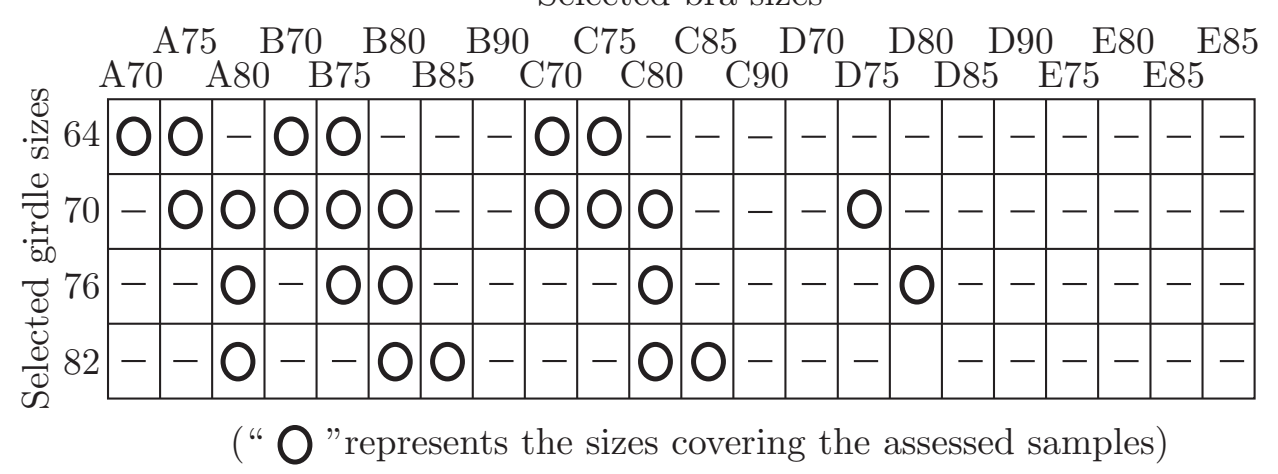

Fig. 1: Matching information of size groups and the current samples.

The reverse engineering software was used to patch the 3D scanning data of the 75 samples. The 3D data was processed into the rotatable dynamic clips, with 3D images transformed into 2D front and side view images. Faces of the subjects were blurred to reduce any possible interference to the assessment process, so as to make preparation for further assessment.

\subsection{Subjective Assessment}

In order to more conveniently observe the body shape features and breast features of the assessed samples, the research team developed Assessment System of Body Beauty as the software platform for subjective assessment, and the input of the rotatable clips, 2D images and questionnaire results were used to assist judges in assessing the breast shape beauty and the whole body beauty of the samples.

The major characteristics of the assessment system are as follows: 1) every judge can establish his or her own database to ensure finishing assessment of all the subjects; 2) judges can randomly select samples from the database to conveniently observe and assess breasts through 3D clips and 2D images; 3) one questionnaire is only for one sample, and results of the questionnaire can be saved into the database automatically; 4) the system can display the current sample, last sample and next sample at the same time, and validity of assessment data can be controlled through browsing, comparison and repeated confirmations; 5) the system can magnify any part of the samples and hence the detailed observation can be realized.

The breast beauty assessment system sets 12 questions related to breast shape beauty and the whole body shape beauty. Five choices, 1, 3, 5, 7 and 9, were available for judges, namely 5 scales 
from very ugly to very beautiful, with 5 as the intermediate scale. The assessment questions involved the whole body shape, the whole body front and side contour, the breast front view and side view, chubbiness of the breast, erectness of the breast, distance between two bust points, height of the breast (in proportion to the whole body height), profile line a, b of the breast, beauty degree of $\mathrm{a}+\mathrm{b}$ (shown as Fig. 2), symmetry of breasts, and so on.

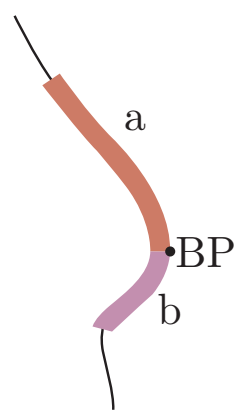

Fig. 2: Side curve of the breast for assessment

\subsection{Anthropometric Measurements}

To better assess breast shapes and explore the relationship between the subjective breast shape beauty assessment results and the breast shapes, this study integrated the manual method with 2D contour measurements and the 3D scanning method (see Fig. 3), and selected 64 body measurement items related to the breast and whole body shape, including 7 items on height, 8 items on width, 7 items on thickness, 5 items on girth, 6 items on body surface length, 16 items on breast, 7 items on body surface angle, 3 items on volume, 5 items on weight \& thickness of fat and others. Besides, some indirect calculation items were set so as to better describe the breast shape features, including ratio of head length to body height, ratio of breast height to body height, difference between bust girth and underbust girth, compressed ratio of the breast, UBL compressed ratio, ratio of width to depth of the breast, and so on, with a total of 70 measurements.

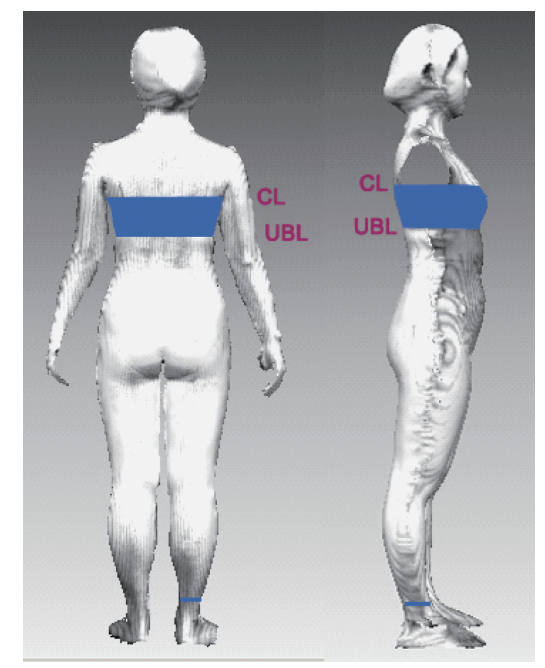

Fig. 3: Chest area volume measuring by 3D body scan data 


\section{Results and Discussion}

\subsection{Subjective Assessment Results \& Discussion}

\subsubsection{Subjective Beauty Assessment Results}

Out of the overall assessment results on the 75 assessed samples given by the 43 judges, 13 questions related to the breast beauty and whole body shape beauty has gained the following statistical results as shown in Table 1. Among them, the assessment items on detailed assessment of the breast, "distance of the bust points" and "height of the bust point (as against the whole body height)" have obtained the highest mean scores, 6.76 and 6.75; proportions of "appropriate" for them were also quite high, respectively $53.4 \%$ and $56.7 \%$. The item "chubbiness of the breast (in proportion to the whole body)" (6.02) followed, with the proportion of "appropriate" $42.2 \%$. The item, most close to the middle scale 5 out of assessment scales 1-9, was "symmetry of the two breasts" (4.95), with the proportion of "beautiful" \& "quite beautiful" 32.5\%. Items with quite low beauty assessment mean scores include "breast front view beauty" (3.99), "breast side view beauty" (3.99), "profile line a of the breast" (3.81) and "profile beauty of the whole body" (3.99); for them, the proportion of "beautiful" and "quite beautiful" was relatively low, respectively $16.0 \%, 14.3 \%, 13.4 \%$ and $15.7 \%$, and they also saw the similar CV results. The CV values of the four items were relatively high among the 13 assessment items, which comparatively indicated large dispersion of assessment scores. Assessment results revealed that items with a little relevance with body curve, such as "distance of bust points", "chubbiness of the breast (in proportion to the whole body)" and "height of the bust point (as against the whole body height)", have witnessed relatively a high beauty degree of the breast. For the large individual difference, items like "profile of the whole body", "breast front view" and "profile line of the breast" have obtained the low

Table 1: Descriptive statistics summary of subjective beauty assessment from questionnaires

\begin{tabular}{lcccc}
\hline Assessment Items & Mean & Std. Deviation & $\begin{array}{c}\text { Proportion of beautiful } \\
\text { \& quite beautiful (\%) }\end{array}$ & CV (\%) \\
\hline Whole body shape beauty & 4.16 & 2.02 & 16.7 & 48.6 \\
Whole body front contour beauty & 4.25 & 2.04 & 18.2 & 47.9 \\
Whole body profile beauty & 3.99 & 2.03 & 15.7 & 50.9 \\
Breast front view beauty & 3.99 & 2.10 & 16.0 & 52.7 \\
Breast side view beauty & 3.99 & 2.03 & 14.3 & 50.9 \\
$\quad$ Chubbiness of the breast & 6.02 & 2.89 & 42.2 & 48.0 \\
(in proportion to the whole body) & & & 26.4 & 53.0 \\
Erectness of the breast & 4.52 & 2.40 & 53.4 & 39.0 \\
Distance of bust points & 6.76 & 2.64 & 56.7 & 42.2 \\
$\quad$ Height of the bust point & 6.75 & 2.85 & 13.4 & 54.0 \\
(as against the whole body height) & & & 21.0 & 49.7 \\
a profile line of the breast & 3.81 & 2.06 & 14.9 & 50.2 \\
b profile line of the breast & 4.30 & 2.14 & 32.5 & 50.2 \\
a+b profile line of the breast & 4.07 & 2.04 & 2.49 & \\
Symmetry of the breast & 4.95 & &
\end{tabular}


percentage of good curve assessment results.

\subsubsection{Correlation Analysis}

To investigate the relationship between the breast shape beauty and the whole body shape beauty, the Pearson correlation analysis of questionnaire scores of the 13 questions was conducted. Correlation coefficients above or close to 0.8 indicate the high correlation between scores of two beauty assessment items. Correlation coefficients above 0.5 but below 0.8 indicate a certain correlation existing between two assessment items and with the same changing tendency of the scores. The results are explained as follows:

1) High correlation exists between the whole body shape beauty and such items as whole body front contour beauty (0.885) and whole body profile beauty (0.770); a certain correlation exists between the whole body shape beauty and such items as breast front view (0.611) and breast side view (0.524).

2) There is quite a high correlation between the whole body front contour beauty and whole body profile beauty with coefficient 0.717 ; meanwhile, the assessment scores of both are correlated to the breast front view and breast side view to a certain degree.

3) Quite high correlation exists between the breast front view beauty (Q04) and items such as breast side view, erectness of the breast, profile line a of the breast, profile line $\mathrm{b}$ of the breast and profile line $\mathrm{a}+\mathrm{b}$ of the breast, with coefficients respectively $0.752,0.606,0.609,0.613$ and 0.635 .

4) Similar to the beauty assessment results on the breast front view, a relatively high correlation also exists between the breast side view beauty and items such as breast front view, erectness of the breast, profile line a of the breast, profile line $\mathrm{b}$ of the breast and profile line $\mathrm{a}+\mathrm{b}$ of the breast, with coefficients respectively $0.752,0.582,0.716,0.731$ and 0.754 .

5) Regarding the correlation between items related to the detailed description of the breast, there is a certain correlation between erectness of the breast and items related to the breast profile lines such as profile line $\mathrm{a}, \mathrm{b}$ and $\mathrm{a}+\mathrm{b}$ with coefficients respectively $0.613,0.582$ and 0.613 ; the weak correlation or nearly zero correlation is present between items such as chubbiness of the breast (in proportion to the whole body), distance between two bust points, height of the bust point (as against the whole body height) and symmetry of the breast, and other assessment items.

From the above analysis, it can be concluded that beauty degree of the breast profile lines directly affects breast front view and side view beauty, and the breast front view also exerts influence on assessment results of the whole body front contour, whole body profile and whole body shape beauty.

\subsection{Exploration of Breast Shape Beauty Based on Anthropometric Data}

This section focuses on discussion of the relationship between the breast front and side view beauty and the relevant anthropometric data. After testing the abnormal values of measurements data of the 75 assessed subjects and eliminating missing values beyond $\pm 4 \mathrm{SD}$ (standard deviation) [21], statistical analysis software was used to complete the fundamental statistical analysis of the 70 anthropometric measurements. According to the statistical mean values and standard deviation of "Q3 breast front view beauty" and "Q4 breast side view beauty" in the subjective 
beauty assessment, the assessment data was divided into 3 sections, respectively the middle part possessing $30 \%$ of the data, the top part and the lower part. In the following formula (1), $\mu$ represents mean value, $\sigma$ refers to standard deviation, and with the standard regular distribution chart, range of $z_{i}$, the middle $30 \%$ part, was determined as \pm 0.39 , with 0.39 as the boundary value. Among the 75 subjects, 13 subjects have seen their assessment scores of Q3 and Q4 falling into the top part beyond the middle $30 \%$, and their breast shapes were regarded as beautiful. Among the 13 subjects, there are 3 aged 20-24, 9 aged 25-29 and 1 at the age of 30-34.

$$
X i=\mu+\sigma z_{i}
$$

Pearson correlation analysis results indicated that the assessment scores of "Q3 breast front view beauty" and "Q4 breast side view beauty" had mainly negative relevance with major width and thickness measurements, girth measurements and detailed breast measurements on the torso. Based on the anthropometric data of the 75 assessed subjects, Table 2 lists the basic statistical results of width, thickness and girth items related to breast and whole body shape beauty (see Fig. 4). In order to better observe the difference between measurements of subjects assessed as beauty and the mean values of the whole subject group, mean values of measurements of 13

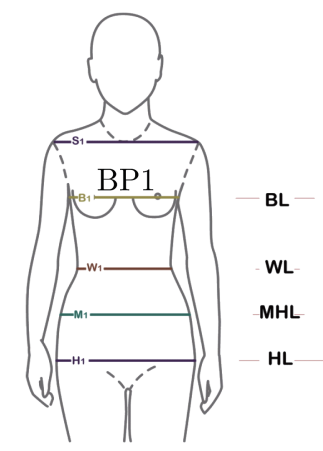

(a) Width items

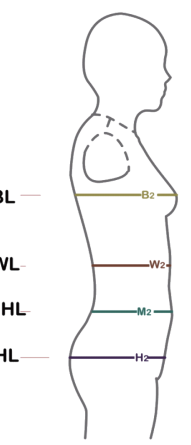

(b) Thickness items

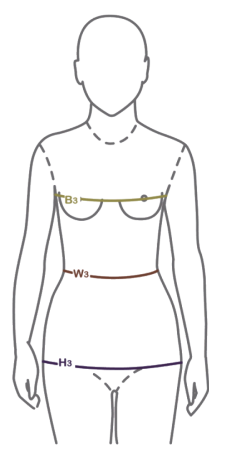

Fig. 4: Width, thickness and girth items related to breast and whole body shape beauty

Table 2: Descriptive statistics summary of measurements related to breast and whole body shape beauty

\begin{tabular}{lcccccc}
\hline Measurements & $\begin{array}{c}\text { Mean (mean of } \\
\text { beauty subjects, cm) }\end{array}$ & $\begin{array}{c}\text { Std. D. } \\
(\mathrm{cm})\end{array}$ & $\begin{array}{c}\text { Min. } \\
(\mathrm{cm})\end{array}$ & $\begin{array}{c}\text { Max. } \\
(\mathrm{cm})\end{array}$ & $\begin{array}{c}\text { CV } \\
(\%)\end{array}$ & $\begin{array}{c}\text { Ratio (ratio of } \\
\text { beauty subjects) }\end{array}$ \\
\hline S1: Shoulder width 2 & $32.8(\mathbf{3 3 . 0})$ & 1.7 & 28.4 & 36.3 & 5.9 & $1.29(\mathbf{1 . 3 4})$ \\
B1: Bust width & $28.0(\mathbf{2 7 . 3})$ & 1.5 & 24.7 & 32.3 & 5.3 & $1.10(\mathbf{1 . 1 0})$ \\
BP1: Bust point width & $19.5(\mathbf{1 8 . 6})$ & 1.5 & 15.8 & 22.7 & 7.6 & $0.77(\mathbf{0 . 7 5})$ \\
W1: Waist width & $25.4(\mathbf{2 4 . 7})$ & 1.8 & 20.0 & 29.0 & 7.0 & $1(\mathbf{1})$ \\
M1: Abdomen width & $31.4(\mathbf{3 0 . 6})$ & 1.9 & 26.6 & 36.0 & 6.2 & $1.24(\mathbf{1 . 2 4})$ \\
H1: Hip width & $33.7(\mathbf{3 3 . 3})$ & 1.4 & 30.0 & 36.7 & 4.1 & $1.33(\mathbf{1 . 3 5})$ \\
B2: Bust depth & $23.1(\mathbf{2 2 . 5})$ & 1.6 & 20.0 & 26.8 & 7.1 & $1.20(\mathbf{1 . 2 3})$ \\
W2: Waist depth & $19.2(\mathbf{1 8 . 2})$ & 2.0 & 15.3 & 23.6 & 10.3 & $1(\mathbf{1})$ \\
M2: Abdomen depth & $22.0(\mathbf{2 0 . 7})$ & 2.2 & 17.8 & 27.5 & 10.2 & $1.15(\mathbf{1 . 1 4})$ \\
H2: Hip depth & $23.0(\mathbf{2 2 . 0})$ & 1.8 & 19.2 & 28.2 & 7.7 & $1.20(\mathbf{1 . 2 1})$ \\
B3: Bust girth & $90.0(\mathbf{8 8 . 8})$ & 5.0 & 80.5 & 100.9 & 5.5 & $1.25(\mathbf{1 . 2 9})$ \\
W3: Waist girth & $71.9(\mathbf{6 9 . 1})$ & 5.6 & 62.6 & 82.0 & 7.7 & $1(\mathbf{1})$ \\
H3: Hip girth & $92.1(\mathbf{9 0 . 1})$ & 4.3 & 83.4 & 102.6 & 4.6 & $1.28(\mathbf{1 . 3 0})$ \\
\hline
\end{tabular}


subjects with relatively beautiful breast shapes were listed in brackets of column 2 and column 7 . Meanwhile, in column 7, mean values of width, thickness and girth of the waist were supposed as 1 , so as to obtain the proportion of width, thickness and girth of other parts to that of the waist.

Comparison results indicated that in contrast with the whole assessed subjects, subjects with relatively beautiful breast shapes have the following features: 1) on width measurements (in column 2), all items including bust width, bust point width, waist width, abdomen width and hip width, had relatively small values, except shoulder width, had the relatively larger values. From the aspect of proportion in column 7, the 13 beautiful subjects tended to possess slimmer waist and wider shoulder and hip, with smaller bust point width. 2) on thickness items, for subjects with beautiful breast shapes, actual measurement values of 4 items were all smaller than the average values of the whole subject group, especially waist depth and abdomen depth. In regard to proportions (in column 7 ), breasts $(1: 1.23 \mathrm{Vs} 1: 1.20)$ and hips $(1: 1.21 \mathrm{Vs}$ 1:1.20) of those beautiful subjects were more protruding, and their abdomen (1:1.14 Vs 1:1.15) were much flatter, with relatively more attractive profile curves and 3D shapes. 3) on girth items, mean values of bust girth, waist girth and hip girth of those subjects with much more beautiful breast shapes were all smaller than the whole average values, but on items of proportion, more attractive curves were reflected, with relatively more chubby breasts (1:1.29 Vs 1:1.25) and hips (1:1.30 Vs 1:1.28).

Measurements diagrams and basic statistical results of major breast measurement items related to breast shape beauty are shown in Fig. 5 and Table 3. Those measurement items include 3 body surface length items, S-B, F-RB \& F-LB, 3 beeline measurement items (AB, AC \& BC) and the corresponding 3 angles around Front Neck Point (FNP), Right Bust Point (RBP) and Left Bust Point (LBP). Same as Table 3, measurements mean values of the 13 subjects with beautiful breast shapes are given in bold in brackets of column 2 and column 7 in Table 3. Comparison results indicate that the average height of the whole subjects is $158.5 \mathrm{~cm}$, the average height of the beautiful subjects is $159.8 \mathrm{~cm}$, but on the 6 measurement items of S-B, F-RB, F-LB, AB, AC \& $\mathrm{BC}$, their average values are all smaller than the whole average values, which indicates that the beauty criteria for the breast shape is convergence and erectness of the breast with the triangle formed by FNP, RBP \& LBP being isosceles triangle close to the equilateral triangle.

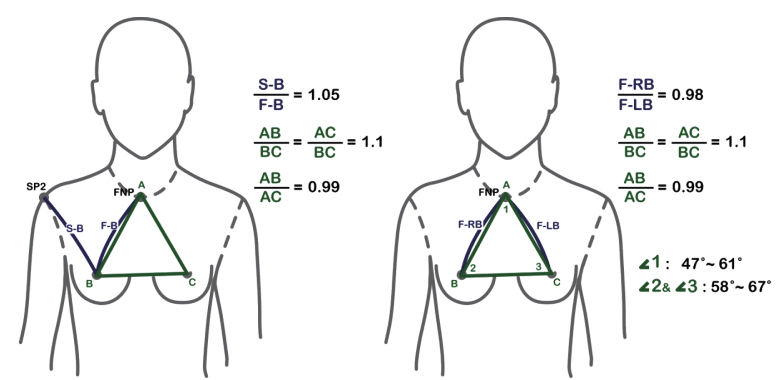

Fig. 5: Detailed breast measurement items related to breast shape beauty (based on 13 subjects with quite beautiful breasts).

The determinant items for breast sizes, relating to breast shape beauty, were investigated, including underbust girth, difference between bust girth and underbust girth and the breast depth width ratio. Results are shown as follows: the ratio of bust height to whole body height was 0.71 ; underbust girth of all the subjects ranged from $68.3 \mathrm{~cm}$ to $87.3 \mathrm{~cm}$, while under bust girth of the 13 subjects with quite beautiful breast shapes ranged from $70.6 \mathrm{~cm}$ to $80.05 \mathrm{~cm}$; difference between bust girth and under bust girth of all the subjects was at $8.7-18.5 \mathrm{~cm}$, while difference between bust girth and under bust girth of subjects with beautiful breast shapes was 
Table 3: Descriptive statistics summary of detailed breast measurements related to breast shape beauty

\begin{tabular}{lcccccc}
\hline Measurements & $\begin{array}{c}\text { Mean }(\text { mean } \\
\text { of beauty } \\
\text { subjects, cm) }\end{array}$ & $\begin{array}{c}\text { Std. D. } \\
(\mathrm{cm})\end{array}$ & $\begin{array}{c}\text { Min. } \\
(\mathrm{cm})\end{array}$ & $\begin{array}{c}\text { Max. } \\
(\mathrm{cm})\end{array}$ & $\begin{array}{l}\text { CV } \\
(\%)\end{array}$ & $\begin{array}{c}\text { Ratio (ratio of } \\
\text { beauty subjects) }\end{array}$ \\
\hline S-B: Surface length from SP2 to right BP & $22.4(\mathbf{2 1 . 7})$ & 2.2 & 18.3 & 30.2 & 9.7 & S-B/F-RB \\
F-RB: Surface length from FNP to right BP & $21.3(\mathbf{2 0 . 7})$ & 1.9 & 17.7 & 26.8 & 9.1 & $1.06(\mathbf{1 . 0 5})$ \\
F-LB: Surface length from FNP to left BP & $21.7(\mathbf{2 1 . 1})$ & 2.0 & 18.4 & 28.0 & 9.3 & F-RB/F-LB:0.98(0.98) \\
AB: Straight length from FNP to right BP & $21.2(\mathbf{2 0 . 6})$ & 1.8 & 17.7 & 25.9 & 8.3 & AB/BC: $1.09(\mathbf{1 . 1 1})$ \\
AC: Straight length from FNP to right RP & $21.6(\mathbf{2 1 . 0})$ & 1.8 & 18.4 & 26.3 & 8.5 & AC/BC:1.11 (1.13) \\
BC: Bust point width & $19.5(\mathbf{1 8 . 6})$ & 1.5 & 15.8 & 22.7 & 7.6 & \\
Angle 1 of the breast triangle $\left(^{\circ}\right)$ & $54(\mathbf{5 3})$ & 4.9 & 40.5 & 65.9 & 9.1 & \\
Angle 2 of the breast triangle $\left(^{\circ}\right)$ & $64(\mathbf{6 4})$ & 3.3 & 55.9 & 74.4 & 5.2 & \\
Angle 3 of the breast triangle $\left(^{\circ}\right)$ & $62(\mathbf{6 3})$ & 2.8 & 55.6 & 68.6 & 4.5 & \\
\hline
\end{tabular}

at $11.4-17.2 \mathrm{~cm}$; the range of the breast depth width ratio of all the subjects was around $0.23-$ 0.54 , while the compressed ratio of the breast of those subjects with beautiful breasts was around 0.33-0.45. The comparison results on data distribution of the three measurement items indicated that subjects with beautiful breasts possessed an appropriated thorax and their breasts were also appropriately chubby.

\subsection{Establishment of the Regression Equation}

To further investigate the relationship between the assessment scores of the breast shape beauty and the measurements relevant to the breast, the waist and other body parts, "the beauty assessment score of the breast side view", which can better reflect the breast curve and was highly correlated to the anthropometric data, was selected as a response, the 70 body measurements in current study were used as predictors, and a stepwise regression model was used to get rid of irrelevant predictors to the response. When p-values of the estimated coefficients of predictors was 0.000 , it indicated that those predictors were significantly correlated to the response "beauty assessment score of the breast side view". Therefore, a multiple regression model was matched for response with the significant predictors.

It was revealed from the match results that the adjusted coefficient of multiple determinations (adjusted $\mathrm{R}^{2}$ ) of the dependent variable was 0.606 . From the residual plots, it was also shown that results of the dependent variable were undoubtedly passable on the premise of independence, constant variance and normality $[22,23]$.

In light of the fit results, the regression equation for the dependent variable "the beauty assessment score of the breast side view" can be written as follows:

$$
y=11.974+0.172 x_{1}-0.452 x_{2}-0.064 x_{3}+0.0 .364 x_{4}+0.163 x_{5}
$$

where $y$ is "the beauty assessment score of the breast side view", $x_{1}$ is difference between bust girth and underbust girth, $x_{2}$ is length from front neck point to left bust point, $x_{3}$ is orientation of the breast (Angle), $x_{4}$ is horizontal distance from lower-most point of the breast to bust point, $x_{5}$ is horizontal distance from front waist centre to bust point. 


\section{Conclusion}

The study on relationship between breast and body shape beauty and anthropometric data will be of far-reaching significance for intimate apparel mannequin research and development and bra design on the premise of beauty. Based on 3D scan data, the subjective questionnaire assessment approach was adopted to judge the beauty scale of subjects' breast and body shapes, and then the relationship between the beauty assessment score of the breast shape and the objective anthropometric data was discussed. Research results are concluded as follows:

1) Correlation of beauty assessment scores of breast front view and side view is obvious. The beauty of breast profile curves and breast erectness can directly influence breast front view and side view beauty, and breast front view can also directly affect the assessment scores of whole body front contour, profile and shape beauty.

2) Assessment results on breast and whole body shape beauty indicated that the assessment scores of such items weakly correlated with body curves as "distance of bust points", "chubbiness of the breast (in proportion to the whole body)" and "bust point height (as against the body height)" were relatively high, implying that the relevant body parts were beautiful; while for the large individual differences on some items like whole body profile, breast front view and side view and curve a of the breast profile, the assessment scores of those items were quite low with relatively large dispersion.

3) Compared with other subjects, the width and thickness ratios of those subjects assessed as beautiful tended to have slimmer waist, wider shoulder and hip, more erect and close bust points. Meanwhile, the triangle formed by FNP, RBP \& LBP was an isosceles triangle with the vertex angle slight smaller than $60^{\circ}$.

4) According to the beauty assessment results of breast side view profile, useful empirical equation was established for predicting "the beauty assessment score of the breast side view" from the five measurements, including difference between bust girth and underbust girth, length from front neck point to left bust point, orientation of the breast (Angle), horizontal distance from lower-most point of the breast to bust point, horizontal distance from front waist centre to bust point.

However, there still existed some limitation in the study, which involves the following aspects: the judges were limited to people working in the underwear industry; the breast beauty assessment results obtained from industry judges may be different from general population; the number of the assessed subjects was relatively limited; judges may be affected by media images in their assessment; accountability of the linear regress formula for "the beauty assessment score of the breast front view" was only 0.606. In future studies, the range of assessed subjects will be further expanded, and judges from varied backgrounds will be introduced, in hope of more effective research results being gained through new study methods and new assessment approaches.

\section{Acknowledgements}

The paper is financially supported by the research projects (with grant numbers KZ200910012001 \& PHR201006122) of The Beijing Municipal Commission of Education foundation. 


\section{References}

[1] Wacoal Corp. Golden Canon [M]. Japan: Published Internal Document, 1995

[2] Liang S Z, Zhang X, Zhou J. Basic breast shapes of female undergraduate in the west of China based on 3-D body scanning. Journal of Textiles Research 2007, 28(8), 75-78

[3] GU L, CHEN X R. A research on the curves of the bosom, waist and hip of young females and the sorting process. Knitting Industries 2007, 8, 27-29

[4] LIU L G, SONG R Y. Breast Mastic Smgery. Beijing: Publishing Company of Science, 1995

[5] ZHANG X M. Chinese Beauty. Beijing: Publishing Company of Xinhua, 2005

[6] FANG Z L, Jiang S Z. Aesthetics of Human body. Beijing: Publishing Company of Beijing 2000

[7] HOU Z E, QIN H Z, LAO L J. The research on female breast of north China. Chinese Journal of Plastic Surgery, 1994, 10(2), 66-68

[8] CHEN Z F, WANG H, WU H L. Measurement on breasts of 100 female college students. Medical Journal of Chinese Armed Police Forces 1999, 8(2), 95-98

[9] Westreich, Melvyn M D. Anthropomorphic breast measurement: Protocol and results in 50 women with aesthetically perfect breasts and clinical application. Plastic and Reconstructive Surgery, 1997, 100, 468-479

[10] Catanuto G, Gallo G, Farinella G M, et al. Breast shape analysis on three dimensional models. In Proceedings of Plastic and Reconstructive Surgery of the Breast: Third European Conference 2005

[11] Hogarth B. Dynamic Figure Drawing. Nanning: Guangxi Fine Arts Publishing House, 1997

[12] Barcsay J. Anatomy for The Artist. Beijing: China Youth Press, 2003

[13] DAN N. The Art of Human Body. Beijing: Publications of the Unity Press, 2007

[14] Furnham A, Tan T, McManus C. Waist-to-hip ratio and preferences for body shape: a replication and extension. Personal and Individual Differences 1997, 22(4), 539-549

[15] Henss R. Waist-to-hip ratio and attractiveness: A replication and extension. Personal and Individual Differences 1995, 19, 479-488

[16] Tovée M J, Cornelissen P L. Female and male perceptions of female physical attractiveness in front-view and profile. British Journal of Psychology 2001, 92, 391-402

[17] Fallon A E, Rozin P. Sex differences in the perception of desirable body shape. Journal of abnormal psychology 1985, 94, 102-105

[18] Rozin P, Fallon A E. Body-image, attitudes to weight, and misperceptions of figure preferences of the opposite sex - a comparison of men and women in two generations. Journal of abnormal psychology 1988, 97, 342-345

[19] FZ/T 73012-2004. Brassiere. Beijing: Standards Press of China, 2004

[20] FZ/T 73011-2004. Knitted Girdle. Beijing: Standards Press of China, 2004

[21] Research Institute of Human Engineering for Quality Life. Japanese body size data 1992-1994. Osaka: HQL, 1997

[22] Montgomery, D C. Introduction to Statistical Quality Control (4th Edition). New York: John Wiley \& Sons, 2001

[23] Montgomery, D C. Design and Analysis of Experiments (6th Edition). Hoboken, NJ: Wiley, 2005 